\title{
Differential Expression and Functional Analysis of High-throughput Sequencing about Long Noncoding RNAs in Corneal Transplantation
}

\author{
Jing Wen ${ }^{1}$, Jing $\mathrm{Wu}^{1}$, Huiwen $\mathrm{Tian}^{1}$, Xiaoli $\mathrm{Lu}^{1}$, Shumei Lin ${ }^{1}$, Ke Xiong ${ }^{1}$, Linjiang Chen ${ }^{1}$, \\ Jian $\mathrm{Yu}^{1}$, and Ming $\mathrm{Ma}^{1}$ \\ ${ }^{1}$ Southern Medical University Nanfang Hospital
}

September 17, 2020

\begin{abstract}
Background: Immune rejection is still the main cause of transplant failure of corneal transplantation which mechanism is not fully understood. The purpose of this study is to investigate the differential expression of long noncoding RNAs (lncRNAs) in corneal allograft rejection and to construct a network diagram of the interaction between lncRNAs and microRNAs(miRNAs). Methods: The lncRNAs expression profile of rat corneal transplantation was constructed by high throughput sequencing. The co-expressed mRNA was analyzed by gene ontology (GO), gene and genomic Kyoto encyclopedia (KEGG). An interaction network diagram of lncRNAs, miRNA and rejection related target genes was constructed. Part of the prediction was verified by real-time polymerase chain reaction (qPCR). Results: A total of 285 lncRNAs expressions were detected between the normal group and the autograft group, with 239 lncRNAs significantly upregulated and 46 lncRNAs downregulated, while 162 lncRNAs were upregulated and 20 downregulated between the allograft group and the autograft group. Go and KEGG were used to enrich and analyze the co-expression of mRNA. By analyzing the interaction between lncRNAs, miRNAs and target genes related to corneal allograft rejection, 56 upregulated lncRNAs, 7 downregulated lncRNAs, 6 upregulated miRNAs and 4 downregulated miRNAs were found in allograft and autograft group. Three of the possible pathways were confirmed and verified by qPCR . Conclusions: The results showed that there was a difference in lncRNA expression between normal ,autograft and allograft group. LncRNAs may be a new molecular target in the treatment of corneal injury and corneal allograft rejection by interact with miRNAs.
\end{abstract}

\section{MAIN BODY TEXT}

\section{Introduction}

Corneal transplantation is the most common form of tissue transplantation worldwide. Due to the absence of blood vessels and lymphatic vessels and the lack of local immune cells, cornea is considered to have a natural immunity, which provides a good environment for allograft transplantation. Immune rejection remains the main cause of transplant failure ${ }^{1,2}$. Corneal transplantation has a high success rate in low risk patients. However, for high-risk patients, graft survival decreased significantly even with active systemic use of immunosuppressant ${ }^{3}$. At present, the treatment of corneal transplantation mainly includes steroids, cyclosporine A, tacrolimus, mycophenolate mofetil, rapamycin and other drugs, all of which have extensive side effects ${ }^{4}$. Therefore, it is necessary to further explore the mechanism of corneal transplantation rejection and provide a new direction for the treatment of corneal transplantation rejection. Although the mechanism of inducing immune tolerance in corneal transplantation is varied, the effect is not satisfactory. In recent years, it has been found that long non-coding RNA (lncRNAs) plays an important regulatory role in the immune system. But in corneal transplantation rejection, the role of lncRNAs in regulating immune rejection is unclear. 
lncRNAs refers to RNA molecules whose length is more than 200 nt and does not encode a protein ${ }^{5}$. In recent years, the study of lncRNAs cover epigenetic, transcriptional and post-transcriptional levels, such as chromatin modification, transcriptional activation and inhibition, and the expression of genes that interfere with molecular interference as an inducer of micro-RNA(miRNAs $)^{6}$. However, the functional and biological relevance of most lncRNAs remain unclear. Studies have shown that lncRNAs is a key regulator of gene expression at the transcriptional and posttranscriptional levels in different cell environments and biological processes ${ }^{7,8}$. It is well known that immune cells play an important role in corneal allograft rejection. Many studies have shown that lncRNAs can regulate the homeostasis and function of important immune cells in rejection, such as dendritic cells (DCs), regulatory $\mathrm{T}$ cells (Tregs) and $\mathrm{CD} 8^{+} \mathrm{T}$ cells ${ }^{9-12}$. At the same time, lncRNAs also plays an important role in the inflammatory response when the immune system is activated ${ }^{13}$. In addition, the abnormal expression of lncRNAs has been proved to be involved in the occurrence, development and pathogenesis of lung transplantation, heart transplantation, type 1 diabetes mellitus, rheumatoid arthritis and other transplantation or immune-related diseases, etc. ${ }^{14-19}$ And lncRNAs are expressed in the eyes and are closely related to retinal structure, diabetic retinopathy, corneal neovascularization and other ocular diseases ${ }^{20-24}$. However, the function and mechanism of lncRNAs in corneal allograft rejection is unknown.

The mechanism of lncRNAs are varied, and it can interact with DNA, RNA, protein ${ }^{25}$. LncRNAs can be used as a sponge to inhibit the effect of miRNAs on messenger RNA(mRNAs). In recent years, more and more studies have found that this mechanism plays an important role in the occurrence and development of many diseases. For example, linc-md1 can regulate the expression of MAML1 and MEF2C by combining miR-133 and miR-135 to affect the process of muscle differentiation ${ }^{6}$. And lncRNA H19 inhibits the proliferation and regeneration of intestinal epithelium in ulcerative colitis by inhibiting p53 protein, microRNA 34a and let- $7^{26}$. However, the interaction between lncRNAs and miRNAs in corneal allograft rejection is still unclear.

In this study, the penetrating keratoplasty model was established in rats. The lncRNA expression profiles were analyzed by high-throughput sequencing technique. We compared the differentially expression of lncRNAs between normal group and autogenous group, autogenous group and allogeneic group 14 days after operation. Combined with our previous studies on the differential expression of microRNAs in corneal allograft rejection, we systematically constructed an interaction network of mRNAs, miRNAs, lncRNAs to predict the regulation mechanism related to rejection by integrating the differential expression of miRNAs and mRNAs. Our research shows that lncRNAs play an important role in corneal transplantation and can interact with miRNAs, and suggests that there may be one or more strategies to treat corneal allograft rejection by regulating lncRNAs mediated sponge regulation.

\section{Materials and methods}

\subsection{Animals}

200 220g female Sprague-Dawley (SD) and Wistar rats were obtained from the Animal Experimental Center of Southern Medical University. All the experimental procedures were approved by the Ethical Committee of Animal Research of Southern Medical University. And the feeding and use of laboratory animals and treatment are strictly guided by the statement on the use of Animals in Ophthalmology and Visual Research.

\subsection{Penetrating Corneal Transplantation}

Wistar rats were randomly divided by Random number table method into three groups: normal control group (A), corneal autograft group (B) and corneal allograft transplantation (C). Transplantation method of Model Establishment reference Williams ${ }^{27}$, et al. Autogenous (Wistar-Wistar) and allogeneic keratoplasty (SDWistar) models were established. The general anesthesia of SD, Wistar rats was induced by intraperitoneal injection of $3 \%$ pentobarbital sodium $1.5 \mathrm{ml} / \mathrm{kg}$. Before operation, the conjunctival sac was washed with normal saline and the pupil was fully dilated. The model of allograft transplantation was established. The $3.5 \mathrm{~mm}$ diameter central corneal graft was fixed on the $3.0 \mathrm{~mm}$ diameter implant bed with 8-10 needles sutured by 10-0 thread intermittently to establish corneal allograft model. The model of corneal autograft 
was established by using diameter $3.0 \mathrm{~mm}$ corneal graft, rotated $180^{\circ}$ and then sutured intermittently. The other steps are the same as the above-mentioned corneal allograft model.

If cataracts, severe pre-iris adhesions, or anterior chamber dysplasia occur on the second postoperative day, the corresponding model is removed and supplemented. All models were made by the same surgeon.

Rejection Index

Ten rats in each group were randomly selected to evaluate the survival of postoperative corneal graft. The corneal condition was observed and recorded daily for 21 days, and then three times a week to 100 days. The degree of opacification (0 - 4), edema (0-2) and vascularization (0-4) of corneal graft were evaluated according to the scoring standard of Larkin ${ }^{28}$, and the rejection index (RI) was recorded and calculated. When RI[?]5 and turbid degree of graft [?] 3, it is rejection. If the graft is still transparent at 100 days, it is considered to be long-term survival.

\subsection{Histology}

On the 14th day after operation, each group was selected 5 rats and their operated eyeball was removed. The eyeball was then fixed in $4 \%$ paraformaldehyde solution for no less than 24 hours, then dehydrated and embedded in paraffin, dewaxed at 60 after continuous sectioning at $4 \mu \mathrm{m}$, followed by hematoxylin-eosin (He) staining. The histopathology of the cornea in each group was observed under microscope.

\subsection{Library Construction and Sequencing.}

Each group was selected 6 corneas and total RNA was isolated using the Trizol (Invitrogen) according to the manufacturer's protocol, and ribosomal RNA was removed using the Ribo-Zero kit (Epicentre, Madison, WI, USA).Fragmented RNA (the average length was approximately $200 \mathrm{bp}$ ) were subjected to first strand and second strand cDNA synthesis following by adaptor ligation and enrichment with a low-cycle according to instructions of NEBNext@ Ultra RNA Library Prep Kit for Illumina (NEB, USA). The purified library products were evaluated using the Agilent 2200 TapeStation and Qubit(r)2.0(Life Technologies, USA). The libraries were paired-end sequenced at Guangzhou RiboBio Co., Ltd. (Guangzhou, China)using (2x150 bp) HiSeq3000.

\subsection{Bioinformatics Analysis and Functional Enrichment Analysis}

The clean reads were obtained after removal of reads containing adapter, ploy-N and at low quality from raw data. HISAT2 was used to align the clean reads to the mouse reference genome mm10 with default parameters. HTSeq was subsequently employed to convert aligned short reads into read counts for each gene model. Differential expression was assessed by DEseq using read counts as input. The BenjaminiHochberg multipletest correction method was enabled. Differentially expressed genes were chosen according to the criteria of fold change $>2$ and adjusted p-value $<0.05$. All the differentially expressed genes were used for heat map analysis and Kyoto Encyclopedia of Genes and Genomes (KEGG) ontology enrichment analyses. For KEGG enrichment analysis, a P-value $<0.05$ was used as the threshold to determine significant enrichment of the gene sets.

\subsection{Interaction Between lncRNA/miRNA/mRNA}

Combined with our previous study on the differential expression of miRNA in corneal allograft rejection ${ }^{29}$, four software (TargetScan,Miranda,miRDB,CLIP) were used to predict target genes, select overlapping targets and intersect with significantly altered target genes. The interaction network diagrams of lncRNAs and miRNAs in normal group and autogenic group, autogenic group and allogeneic group were constructed respectively. In addition, by integrating the differential expression of miRNA and target genes, a network map of interaction between lncRNAs, miRNAs and target genes related to corneal allograft rejection was constructed.

2.7 Quantitative reverse transcriptase polymerase chain reaction (qRT-PCR) 
Quantitative reverse transcriptase polymerase chain reaction was used to validate the key differentially expressed lncRNAs, miRNAs and target genes. Total RNA was extracted from 5 normal, 5 isograft, and 5 allograft corneas using Trizol (Takara, Japan) reagent respectively. GAPDH mRNA was used as an endogenous control for mRNA and lncRNAs. U6 was used as an endogenous control for miRNAs. Reverse transcription of the total RNA was performed using PrimeScript RT Master Mix (Takara, Japan) for mRNAs and lncRNAs, and reverse transcription of miRNAs with Mir-X miRNA First-Strand Synthesis (Takara, Japan). qRT-PCR was performed with SYBR Premix Ex Taq II (Takara, Japan) on an Quantstudio5 real-time PCR system. qRT-PCR primers are shown in Table 1 (Table 1 ).

\subsection{Statistical analysis}

Use SPSS 20. 0 software for analysis, statistical analysis data expressed as mean + - standard. The survival map of corneal graft was plotted with GraphPad Prism Version 7.0.The normal distribution of the data set was tested by Kolmogorov-Smirnov. The comparison of mRNA in corneal tissues of each group was performed by One-Way ANOVA test, and the difference was statistically significant $(\mathrm{p}<0.05)$. All experiments are repeated at least three times.

\section{Results}

\subsection{Survival Analysis of Corneal Grafts}

In the study of corneal graft rejection, we found that in different groups of rat eye models, transient edema and opacity of corneal graft appeared 3 days after operation. On the 6th day after operation, neovascularization appeared around corneal suture in corneal autograft group (B) and corneal allograft transplantation (C), and the graft was transparent. On the 9th day after operation, neovascularization appeared at the edge of the corneal graft in group B, but the corneal graft was still transparent. On the 8th day after operation, the corneal transparency, iris texture were decreased and large number of neovascularization appeared in 2 rats in group C. And on the 14th day after operation, all corneal grafts in group C had RI points[?] 5 and opacity degree [?] 3 points (Figure 1 ). In group B, the cornea remained transparent until 100 days with RI pointsi 5 . We analyzed the survival of corneal graft in each group (Figure 2 ).

\subsection{Histological studies}

He staining showed that the cornea in group A was a normal rats corneal section with clear anatomic structure and no neovascularization (Figure 3A, 3a ), B group with a small number of inflammatory cells and neovascularization (Figure 3B, 3b ). In group $\mathrm{C}$, a lot of inflammatory cell infiltration and neovascularization could be seen in the whole layer of corneal tissue (Figure 3C, 3c ), including opacity and edema of corneal graft, disorder of corneal structure and large amount of inflammatory cell infiltration and neovascularization.

\subsection{Differentially expression of lncRNA and mRNAs in the 3 groups}

The sequence data were filtered by volcanic map (Figure $\mathbf{4 A}, \mathbf{4 B}, \mathbf{4 C}$ ) to illustrate the differentially expressed lncRNAs between the three groups. Filter criteria are $\mid \log 2$ (fold change) $\mid[?] 2, \mathrm{p}<0.05$. The expression of lncRNAs in allograft group and auto-transplantation group were significantly different, of which 142 were up-regulated and 20 were down-regulated. There were 285 lncRNAs differential expression in normal group and autograft group, among which 239 were up-regulated and 46 were down-regulated. There were 885 lncRNAs differential expression in normal group and allograft group, among which 644 were up-regulated and 241 were down-regulated.

At the same time, we found a new differential expression lncRNAs. In addition, it was found that the expression of 1097 mRNAs were up-regulated and the expression of 1097 mRNAs were down-regulated between the allograft group and the autograft group, while 2765 up-regulated and 177 down-regulated between the normal group and the autograft group. There were 4887 mRNAs differential expression in normal group and allograft group, among which 3930 were up-regulated and 957 were down-regulated.

3.4Gene Enrichment and Pathway Analysis of the LncRNA Target Genes 
In addition to the differential analysis of lncRNA, we screened 3725 genes and 1749 targeted genes in the autograft group and the normal group, as well as in the autograft group and allograft group. The selected genes were analyzed by Gene Ontology (GO) enrichment and KEGG pathway analysis. The target genes were analyzed by differential analysis.

Go analysis (Figure 5A, 5B, 5C ) involves three fields: Cellular component (Cc), Molecular Function (MF), and Biological Process (BP). "binding", "cell", "cellular process" were the most abundant between normal group and autogenic group. Interestingly, autograft group and allograft group were enriched at most in accordance with the foregoing.

Our KEGG pathway analysis results were shown in figure (Figure $\mathbf{5 D}, \mathbf{5 E}, \mathbf{5 F}$ ). Compared with normal cornea, autograft group has the highest levels of " Metabolic pathways ", " Endocytosis " and " HTLVI infection ". While Autograft and allograft group had the highest levels of " Metabolic pathways ", " Chemokine signaling pathway " and " Rap1 signaling pathway". Moreover, Compared with normal cornea, allograft group has the highest levels of " Metabolic pathways ", " PI3K-Akt signaling pathway ". It is important to note that, $\mathrm{T}$ cell receptor signaling pathway, VEGF signaling pathway is present in both autograft group and allograft group. Metabolic pathways is present in both comparison groups.

3.5Interaction Network of IncRNAs,miRNAs and target genes

In order to further study the relationship between lncRNA and miRNA, target genes, we predicted miRNA target genes by TargetScan, Miranda, miRDB, and CLIP, crossed the differential target genes with lncRNAs, and predicted miRNAs, associated with differential lncRNAs. The interaction between lncRNAs and miRNAs was analyzed (Figure 6A, 6B ). In addition, we found 56 up-regulated lncRNAs and 7 downregulated lncRNAs when the results of autograft group and allograft group were intersected with the target genes associated with corneal allograft rejection. The results showed that 4 down-regulated miRNAs and 6 up-regulated miRNAs, may regulate CD40,Petrc,Pik3cd, Inpp5d, Cd8a, Tnfrsf1b, Nfatc2 (Figure 7 ).

3.6Validation with qRT-PCR

To confirm the interaction between lncRNAs, miRNAs and rejection-related genes, we randomly select three possible pathways , used qRT-PCR to test and verify the relative expression of XR_594048.1,XR_591557.2,XR_595450.2,Ptprc,Pik3cd,Nfatc2,rno-miR-673-5p,rno-miR-384-5p,rno-miR-6331 , using GAPDH and U6 as internal reference respectively, to confirm the co-expression of lncRNAs, miRNAs and target genes in autograft cornea $(\mathrm{n}=5)$, allograft cornea $(\mathrm{n}=5)$ and normal cornea $(\mathrm{n}=5)$. (Figure 8 )

\section{Discussion}

Despite the existence of natural "immunity pardon", which provides a good environment for allograft transplantation, immune rejection is still the main cause of failure of transplantation. Although the mechanisms of inducing immune tolerance during corneal transplantation are varied and there are many immunosuppressive agents, the results are not satisfactory ${ }^{3,30,31}$. In recent years, it has been found that lncRNAs plays an important regulatory role in the immune system, but in corneal allograft rejection, the role of lncRNAs, which regulates immune rejection, is still unclear ${ }^{9,10,13,32}$. Therefore, in this study, the rat models of corneal allograft and corneal penetrating autograft were established. On the 14th day after operation, the corneal grafts in the allograft group all had rejection, and the corneal edema and thickening were obvious in comparison with the other two groups, and the corneal graft in the allograft group was significantly thickened compared with the other two groups, as well as inflammatory cell infiltration and other histological features. We analyzed the differential lncRNAs and its relationship with miRNAs and target genes in corneal transplantation rejection.

The complexity and diversity of RNAs structure and interaction have been revealed in the transcription of several species by high-throughput sequencing (HTS). These methods reveal the important mechanism and biological function of lncRNAs in gene expression and RNA metabolism, as well as in variety diseases 33. The aim of this study was to investigate the changes of lncRNAs in corneal tissues and their interaction with miRNAs and target genes in normal, autograft cornea and allograft corneal tissues by lncRNA 
high-throughput sequencing. The results showed that compared with the autograft group, there were 142 lncRNAs up-regulated and 20 significantly down-regulated in allograft group. We believe that these 162 differentially expressed lncRNAs may be involved in the occurrence and development of corneal allograft rejection. In contrast, compared with normal cornea, 239 lncRNAs were significantly up-regulated and 46 were significantly down-regulated in autograft group. We believe that these 285 lncRNAs may be involved in the mechanism of corneal injury.

Furthermore, we analyzed the target genes of differential lncRNAs by KEGG and GO in three groups. Go analysis involved three areas: Cellular component (Cc), Molecular Function (MF) and Biological Process (BP). Compared normal cornea and autograft cornea, the highest concentrations of KEGG are "Metabolic pathways", "Endocytosis" and "HTLV-I infection". "Metabolic pathways", "Chemokine signaling pathway" and "Rap1 signaling pathway" were the most abundant compared autograft and allograft cornea. "Metabolic pathways" was the highest in the two comparison groups, which might play an important role in both corneal injury and allograft rejection. It is worth noting that compared with the autograft group, the allograft group has a higher concentration of $\mathrm{T}$ cell receptor signaling pathway(TCR) and Vascular endothelial growth factor(VEGF) signaling pathway. TCR stimulation not only activates transcriptional processes, including I $x$ b kinase (Ikk) related NF- $x \mathrm{~B}$ and calcium-dependent NFAT programs, Akt kinase 16 (block 1) inhibits the activity of FOXO family transcription factors. TCR signaling pathway plays an important role in the induction of FOXO3 gene transcription, while FOXO3 inhibits T cell proliferation and induces $\mathrm{T}$ cell apoptosis. In addition, recent studies have shown that TCR plays an important role in the differentiation and function of Treg cells ${ }^{34,35}$.VEGF pathway plays an important role in corneal rejection, and VEGF ligand and receptor family are important regulators of angiogenesis and lymphangiogenesis. The relationship between the degree of vascularization and corneal allograft rejection has long been confirmed ${ }^{36}$. The 14th day after operation, the degree of vascularization and histopathological examination of corneal graft also confirmed that there were more neovascularization on the rejected corneal graft. Previous studies have shown that $\mathrm{T}$ cells can promote angiogenesis and induce corneal allograft rejection in corneal transplantation ${ }^{37}$.

In order to investigate the lncRNAs associated with miRNAs, we analyzed the interaction network of lncRNAs, miRNAs and target genes related to rejection pathway in combination with the results of miRNA high throughput sequencing ${ }^{29}$. It was found that 56 up-regulated lncRNAs and 7 down-regulated lncRNAs, 4 down-regulated and 6 up-regulated miRNAs, maybe regulate Toll-like receptor pathway, cell receptor signaling pathway, TNF signaling pathway and VEGF signaling pathway via regulation of target genes like CD40, Pik3cd, Cd8a, Tnfrsf1b, Nfatc2 and other genes in allograft group and autograft group to regulate the occurrence and development of rejection. We have randomly verified three pairs of interacting lncRNAs and miRNAs, and the related target genes, which are consistent with the predicted results. Among them, Pik3cd is the gene of PI3K. PI3K plays an important role in Toll-like receptor pathway, T-cell receptor signaling pathway, TNF signaling pathway, VEGF signaling pathway, mTOR signaling pathway, and regulates various physiological processes in various diseases. For example, PI3K/mTOR pathway regulates metabolic function and PI3K/AKT pathway promotes endothelial cell angiogenesis ${ }^{38-40}$. Recent studies have shown that miR26b inhibits the PI3K/AKT pathway by directly targeting the gene Pik3cd encoding PI3Ko gene in human T-cell acute lymphoblastic leukemia (T-ALL) cell lines ${ }^{41}$. In conclusion, we think that XR_591557.2 may regulate the occurrence and development of allograft rejection by targeting rno-miR-384-5p and regulating Pik3cd and its related pathways.

In conclusion, high-throughput sequencing of lncRNAs provides a new way to explore the mechanism of corneal transplantation rejection. The differential expression of lncRNAs and bioinformatics analysis are of great significance in elucidating the mechanism of corneal transplantation rejection. The comprehensive analysis of lncRNAs and miRNAs and their target genes provides a feasible scheme for inhibiting corneal transplantation rejection and provides a new idea for the treatment of corneal transplantation rejection. However, further experiments in vivo and in vitro are needed to verify the interaction between lncRNAs and miRNAs in corneal allograft rejection and to determine its targeting relationship.

\section{Acknowledgements:}


Jing Wen: Participated in research design;participated in the performance of the research.

Jing Wu: Participated in research design;participated in the writing of the paper.

Huiwen Tian: Participated in the writing of the paper;participated in data analysis.

Xiaoli Lu: Participated in the performance of the research.

Shumei Lin: Contributed new reagents or analytic tools.

Ke Xiong: Participated in data analysis.

Linjiang Chen:Participated in the performance of the research.

Jian Yu: Participated in literature search.

Ming Ma: Participated in research design;participated in literature search.

\section{Funding:}

This work was funded by: Science and Technology Planning Project of Guangdong Province (2017A020211005); President Funding of Nanfang Hospital (2019B023); President Funding of Nanfang Hospital (2019Z017).

\section{Conflict of Interest:}

The authors declare no conflicts of interest.

\section{References}

1. Armitage WJ, Goodchild C, Griffin MD, Gunn DJ, Hjortdal J, Lohan P, . . . Vabres B. High-risk Corneal Transplantation: Recent Developments and Future Possibilities. Transplantation 2019; 103:2468-78.

2. Alio JL, Montesel A, El Sayyad F, Barraquer RI, Arnalich-Montiel F, Alio Del Barrio JL. Corneal graft failure: an update. Br J Ophthalmol 2020.

3. Yu T, Rajendran V, Griffith M, Forrester JV, Kuffova L. High-risk corneal allografts: A therapeutic challenge. World J Transplant 2016; 6:10-27.

4. Di Zazzo A, Kheirkhah A, Abud TB, Goyal S, Dana R. Management of high-risk corneal transplantation. Surv Ophthalmol 2017; 62:816-27.

5. Guo CJ, Xu G, Chen LL. Mechanisms of Long Noncoding RNA Nuclear Retention. Trends Biochem Sci 2020.

6. Cesana M, Cacchiarelli D, Legnini I, Santini T, Sthandier O, Chinappi M, . . . Bozzoni I. A Long Noncoding RNA Controls Muscle Differentiation by Functioning as a Competing Endogenous RNA (vol 147, pg 358, 2011). Cell 2011; 147:947-.

7. Chen LL. Linking Long Noncoding RNA Localization and Function. Trends Biochem Sci 2016; 41:761-72.

8. Quinn JJ, Chang HY. Unique features of long non-coding RNA biogenesis and function. Nat Rev Genet $2016 ; 17: 47-62$.

9. Mowel WK, Kotzin JJ, McCright SJ, Neal VD, Henao-Mejia J. Control of Immune Cell Homeostasis and Function by lncRNAs. Trends Immunol 2018; 39:55-69.

10. Wang P, Xue Y, Han Y, Lin L, Wu C, Xu S, . . Cao X. The STAT3-binding long noncoding RNA lnc-DC controls human dendritic cell differentiation. Science 2014; 344:310-3.

11. Zemmour D, Pratama A, Loughhead SM, Mathis D, Benoist C. Flicr, a long noncoding RNA, modulates Foxp3 expression and autoimmunity. Proc Natl Acad Sci U S A 2017; 114:E3472-E80. 
12. Sharma S, Findlay GM, Bandukwala HS, Oberdoerffer S, Baust B, Li Z, . . . Rao A. Dephosphorylation of the nuclear factor of activated T cells (NFAT) transcription factor is regulated by an RNA-protein scaffold complex. Proc Natl Acad Sci U S A 2011; 108:11381-6.

13. Chen YG, Satpathy AT, Chang HY. Gene regulation in the immune system by long noncoding RNAs. Nat Immunol 2017; 18:962-72.

14. Dong M, Wang X, Zhao HL, Chen XL, Yuan JH, Guo JY, . . . Li G. Integrated analysis of transcription factor, microRNA and LncRNA in an animal model of obliterative bronchiolitis. Int J Clin Exp Pathol 2015; 8:7050-8.

15. Gu G, Huang Y, Wu C, Guo Z, Ma Y, Xia Q, . . . He X. Differential Expression of Long Noncoding RNAs During Cardiac Allograft Rejection. Transplantation 2017; 101:83-91.

16. Messemaker TC, Frank-Bertoncelj M, Marques RB, Adriaans A, Bakker AM, Daha N, . . Kurreeman F. A novel long non-coding RNA in the rheumatoid arthritis risk locus TRAF1-C5 influences C5 mRNA levels. Genes Immun 2016; 17:85-92.

17. Lu MC, Yu HC, Yu CL, Huang HB, Koo M, Tung CH, Lai NS. Increased expression of long noncoding RNAs LOC100652951 and LOC100506036 in T cells from patients with rheumatoid arthritis facilitates the inflammatory responses. Immunol Res 2016; 64:576-83.

18. Wallace C, Smyth DJ, Maisuria-Armer M, Walker NM, Todd JA, Clayton DG. The imprinted DLK1MEG3 gene region on chromosome 14q32.2 alters susceptibility to type 1 diabetes. Nat Genet 2010; 42:68-71.

19. Taheri M, Eghtedarian R, Dinger ME, Ghafouri-Fard S. Dysregulation of non-coding RNAs in Rheumatoid arthritis. Biomed Pharmacother 2020; 130:110617.

20. Chen W, Yang S, Zhou Z, Zhao X, Zhong J, Reinach PS, Yan D. The Long Noncoding RNA Landscape of the Mouse Eye. Invest Ophthalmol Vis Sci 2017; 58:6308-17.

21. Gong Q, Su G. Roles of miRNAs and long noncoding RNAs in the progression of diabetic retinopathy. Biosci Rep 2017; 37.

22. Jae N, Dimmeler S. Long noncoding RNAs in diabetic retinopathy. Circ Res 2015; 116:1104-6.

23. Krol J, Krol I, Alvarez CP, Fiscella M, Hierlemann A, Roska B, Filipowicz W. A network comprising short and long noncoding RNAs and RNA helicase controls mouse retina architecture. Nat Commun 2015; 6:7305.

24. Huang J, Li YJ, Liu JY, Zhang YY, Li XM, Wang LN, . . . Yan B. Identification of corneal neovascularization-related long noncoding RNAs through microarray analysis. Cornea 2015; 34:580-7.

25. Jandura A, Krause HM. The New RNA World: Growing Evidence for Long Noncoding RNA Functionality. Trends Genet 2017; 33:665-76.

26. Geng H, Bu HF, Liu F, Wu L, Pfeifer K, Chou PM, . . . Tan XD. In Inflamed Intestinal Tissues and Epithelial Cells, Interleukin 22 Signaling Increases Expression of H19 Long Noncoding RNA, Which Promotes Mucosal Regeneration. Gastroenterology 2018; 155:144-55.

27. Williams KA, Coster DJ. Penetrating corneal transplantation in the inbred rat: a new model. Invest Ophthalmol Vis Sci 1985; 26:23-30.

28. Larkin DF, Calder VL, Lightman SL. Identification and characterization of cells infiltrating the graft and aqueous humour in rat corneal allograft rejection. Clin Exp Immunol 1997; 107:381-91.

29. Lu X, Wu J, Ma M, Wu X, Wen J, Yu J. An integrated deep sequencing analysis of microRNAs in transplanted corneas. Mol Immunol 2018; 101:429-39.

30. Niederkorn JY. Corneal transplantation and immune privilege. Int Rev Immunol 2013; 32:57-67. 
31. Niederkorn JY. Immunology of Corneal Allografts: Insights from Animal Models. J Clin Exp Ophthalmol $2015 ; 6$.

32. Sikora M, Marycz K, Smieszek A. Small and Long Non-coding RNAs as Functional Regulators of Bone Homeostasis, Acting Alone or Cooperatively. Mol Ther Nucleic Acids 2020; 21:792-803.

33. Qian X, Zhao J, Yeung PY, Zhang QC, Kwok CK. Revealing lncRNA Structures and Interactions by Sequencing-Based Approaches. Trends Biochem Sci 2019; 44:33-52.

34. Li MO, Rudensky AY. T cell receptor signalling in the control of regulatory $\mathrm{T}$ cell differentiation and function. Nat Rev Immunol 2016; 16:220-33.

35. Luo CT, Li MO. Transcriptional control of regulatory T cell development and function. Trends Immunol 2013; 34:531-9.

36. Cursiefen C, Chen L, Borges LP, Jackson D, Cao J, Radziejewski C, . . . Streilein JW. VEGFA stimulates lymphangiogenesis and hemangiogenesis in inflammatory neovascularization via macrophage recruitment. J Clin Invest 2004; 113:1040-50.

37. Di Zazzo A, Tahvildari M, Subbarayal B, Yin J, Dohlman TH, Inomata T, . . . Dana R. Proangiogenic Function of T Cells in Corneal Transplantation. Transplantation 2017; 101:778-85.

38. Foukas LC, Bilanges B, Bettedi L, Pearce W, Ali K, Sancho S, . . . Vanhaesebroeck B. Long-term p110alpha PI3K inactivation exerts a beneficial effect on metabolism. EMBO Mol Med 2013; 5:563-71.

39. Fruman DA, Chiu H, Hopkins BD, Bagrodia S, Cantley LC, Abraham RT. The PI3K Pathway in Human Disease. Cell 2017; 170:605-35.

40. Zhou Y, Tu C, Zhao Y, Liu H, Zhang S. Placental growth factor enhances angiogenesis in human intestinal microvascular endothelial cells via PI3K/Akt pathway: Potential implications of inflammation bowel disease. Biochem Biophys Res Commun 2016; 470:967-74.

41. Yuan T, Yang Y, Chen J, Li W, Li W, Zhang Q, . . . You MJ. Regulation of PI3K signaling in T-cell acute lymphoblastic leukemia: a novel PTEN/Ikaros/miR-26b mechanism reveals a critical targetable role for PIK3CD. Leukemia 2017; 31:2355-64.

\section{FIGURE LEGENDS}

Figure 1.Corneal morphology in different treatment groups on day 14 after corneal transplantation. (A) Normal group (B) Corneal autograft group (C) Corneal allograft group

Figure 2. Kaplan-Meier survival curve. No corneal rejection occurred in group a and group b, when the corneal survival time in $\mathrm{C}$ group was $(11.0 \pm 2.11) \mathrm{d}$, the difference was statistically significant $(\mathrm{p}<0.001)$.

Figure 3 HE staining of corneal tissue in each group. (A, a) Normal group (B, b) Corneal autograft group $(\mathrm{C}, \mathrm{c})$ Corneal allograft group

Figure 4 Differential expressed lncRNAs between three groups. The volcano plot illustrates the differentially expressed lncRNAs in each group. (A) Normal group VS Autograft group (B) Autograft group VS Allograft group $(\mathrm{C})$ Normal group VS Allograft group

Figure 5 Gene Enrichment and Pathway Analysis of the lncRNA target genes. (A) GO enrichment analysis of differentially expressed genes between normal group and autograft group. (B) GO enrichment analysis of differentially expressed genes between autograft group VS allograft group. (C) GO enrichment analysis of differentially expressed genes between normal group VS allograft group.(D) KEGG enrichment analysis of differentially expressed genes between Normal group and autograft group. (E) KEGG enrichment analysis of differentially expressed genes between autograft group VS allograft group. (F) KEGG enrichment analysis of differentially expressed genes between normal group VS allograft group. 
Figure 6 Interaction network of lncRNAs and miRNAs. (A) Normal group VS Autograft group (B) Autograft group VS Allograft group

Figure 7 Interaction network of lncRNAs,miRNAs and target genes. (A) Normal group VS Autograft group (B) Autograft group VS Allograft group

Figure 8 LncRNAs,miRNAs and genes relative expression difference in three groups. Real-time PCR was used to compare the levels of XR_594048.1,XR_591557.2,XR_595450.2,Ptprc,Pik3cd,Nfatc2,rnomiR-673-5p,rno-miR-384-5p,rno-miR-6331, between Normal group and autograft group ,autograft group and allograft group. GAPDH and U6 was detected as the internal control. Relative gene expression was shown as the fold increase compared with the Normal group(mean+-SD, $\left.{ }^{*} ; 0.05,{ }^{*} \mathrm{P}_{j} 0.01,{ }^{*} \mathrm{P} j 0.001\right)$.

\section{TABLES}

Table 1. Sequence of the primers for qRT-PCR

\begin{tabular}{lll}
\hline Gene name & Forward primer (5'-3') & Reverse primer (5'-3') \\
\hline XR_594048.1 & TGCTACTCCTTCAGACGACTCCAG & TGCTCCGCTCCTCCACAGAAC \\
XR_591557.2 & GCTTCCGAAGTGCAGAATGGTAGG & AACTCCGTGCTCCAGGCTCTC \\
XR_595450.2 & AGCACTCGTGAACGCAGCATC & GCCAGCAAGCATCAACACCAATG \\
Ptprc & GGCAAGGAACAACCGACGATGG & AGCCGTGAGTGTGGTGAGGTC \\
Pik3cd & CTCGGACACCATCGCCAACATC & ACAGTAGCCAGCACAGGAGAGG \\
Nfatc2 & CGGCTCTGCTGTTCTCATGGATG & AATTCCTCCTCTCCTCCTGCTCAC \\
GAPDH & GACATGCCGCCTGGAGAAAC & AGCCCAGGATGCCCTTTAGT \\
rno-miR-673-5p & CTCACAGCTCCGGTCCTTGG & \\
rno-miR-384-5p & cgcgTGTAAACAATTCCTAGGCAATGT & \\
rno-miR-6331 & gCTTTGGTGGCTTAGTTCTTTGTGC & \\
U6 & GGAACGATACAGAGAAGATTAGC & TGGAACGCTTCACGAATTTGCG \\
\hline
\end{tabular}

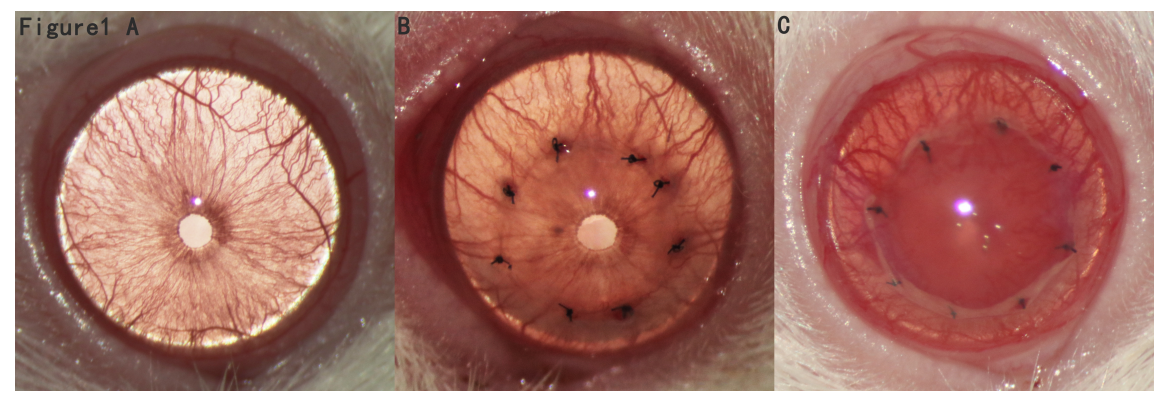



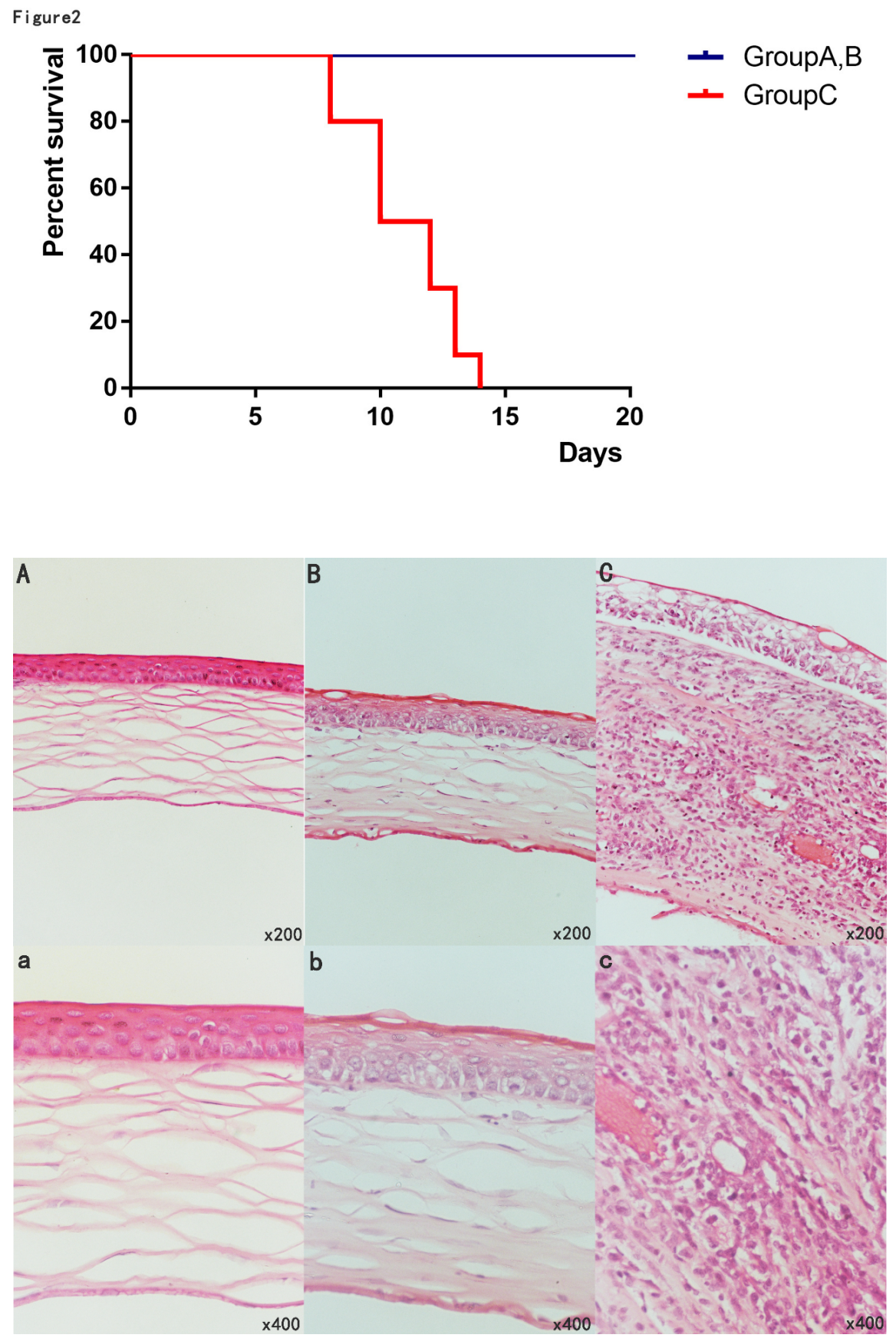


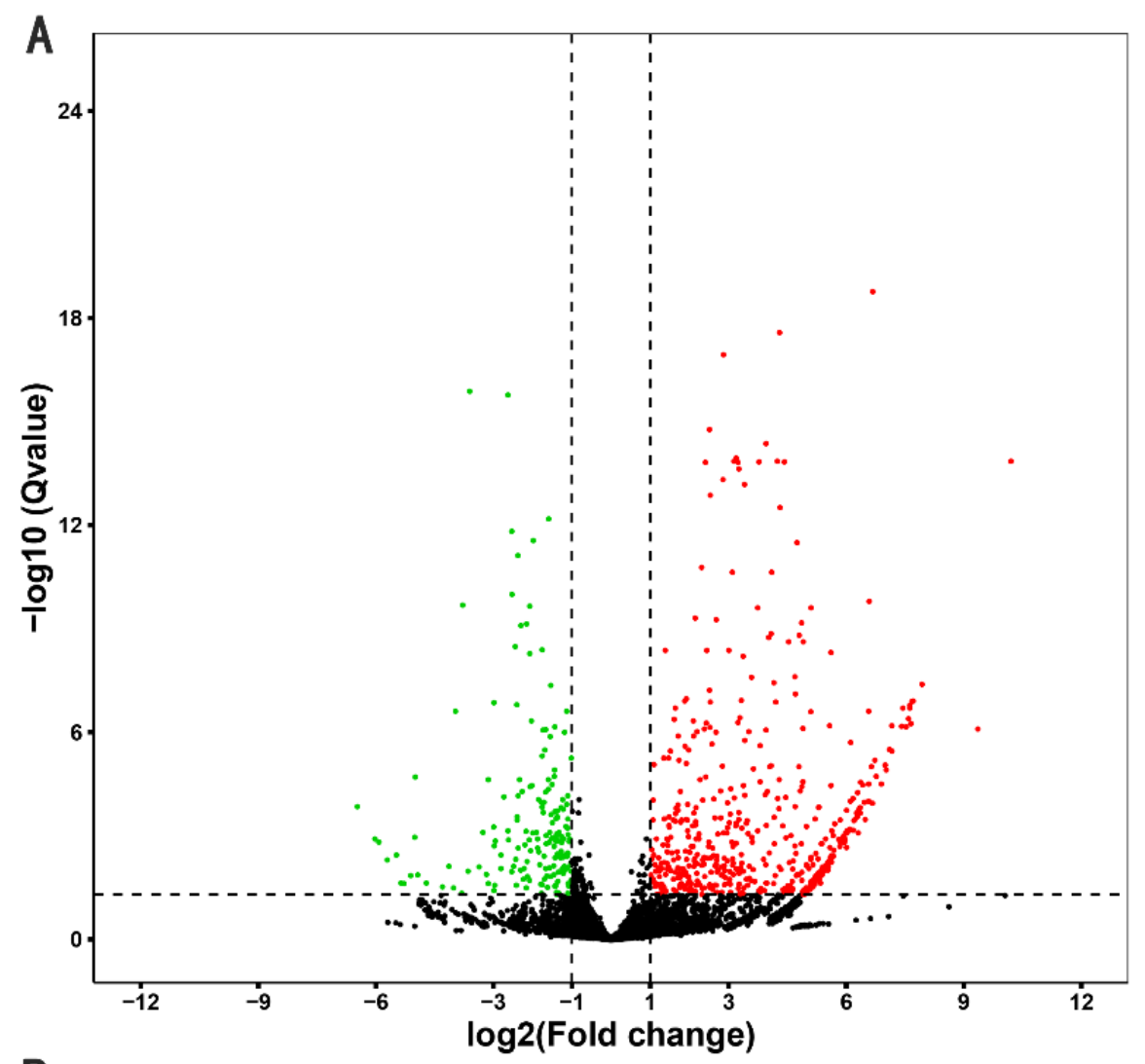

B

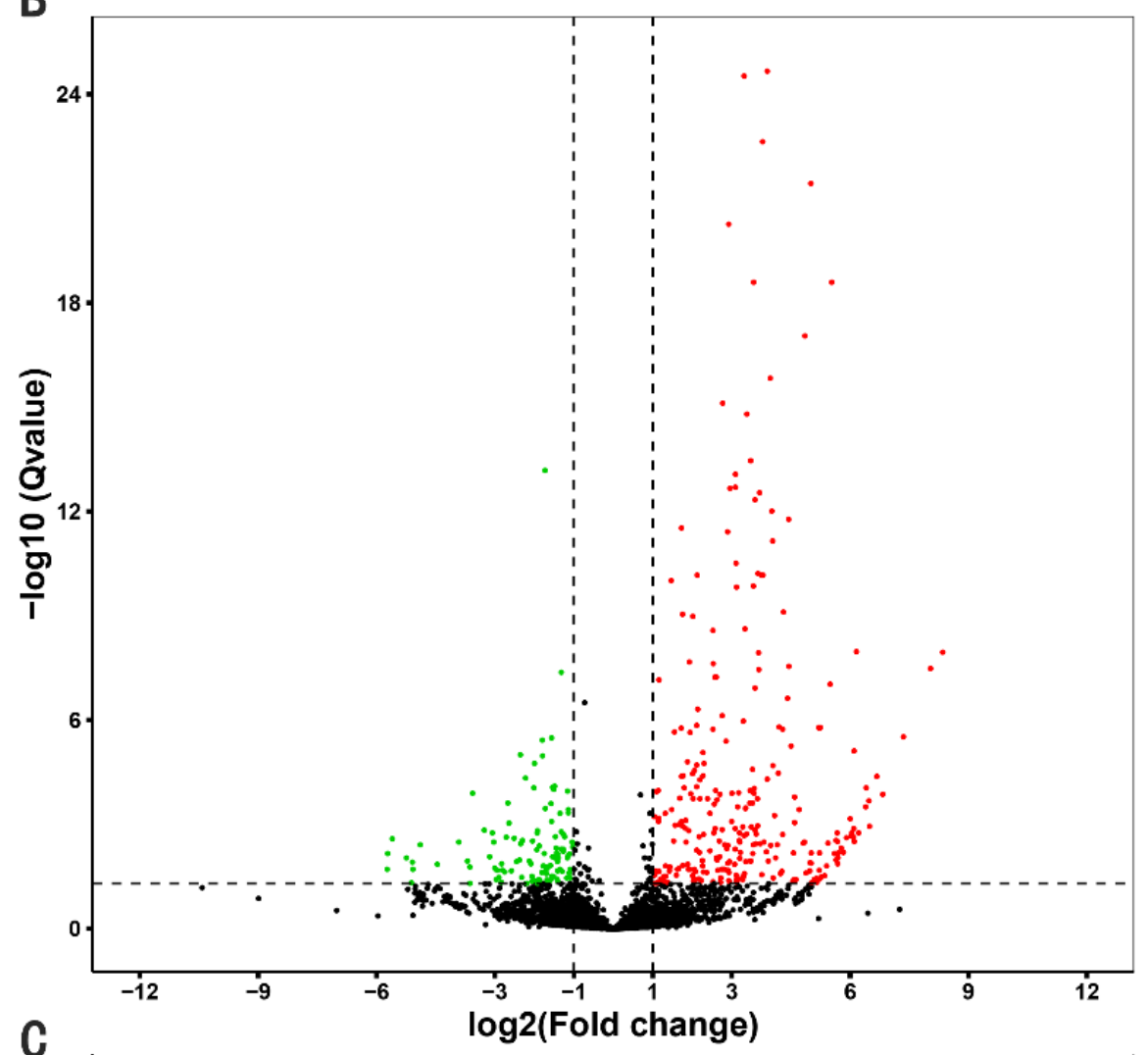

C

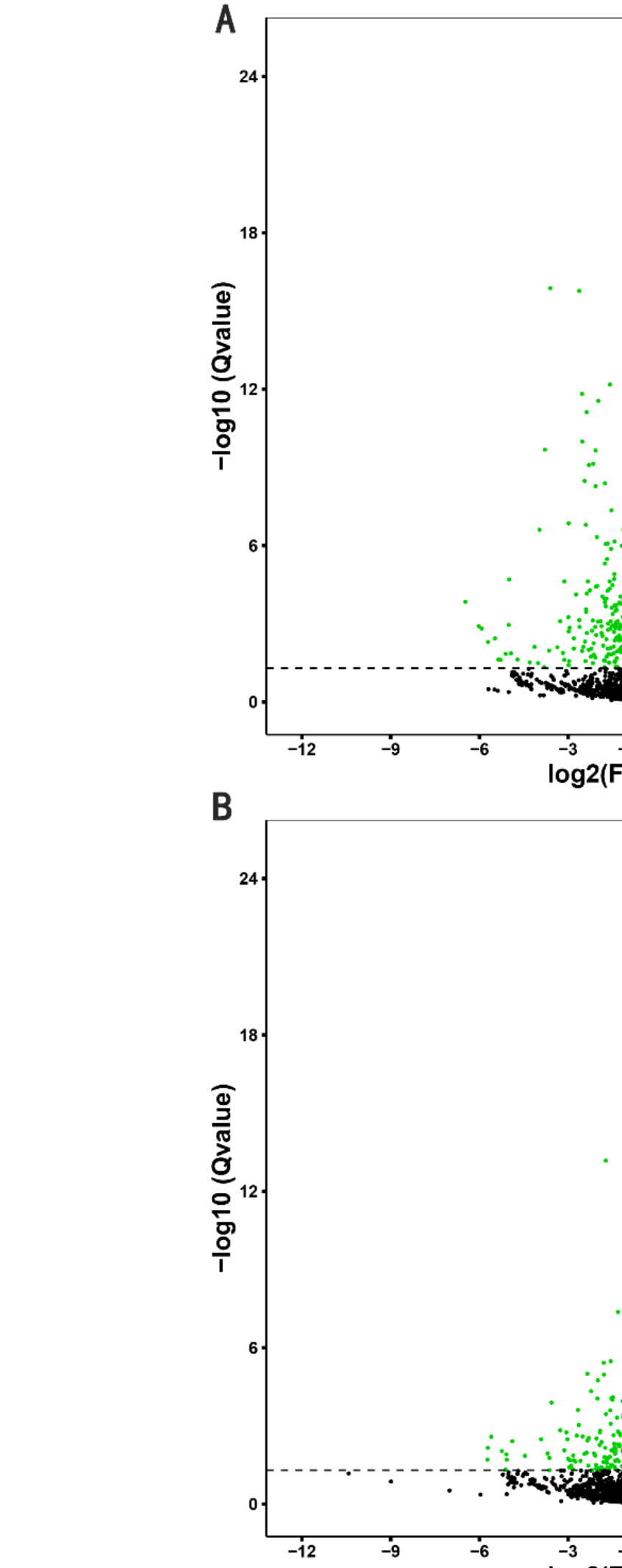



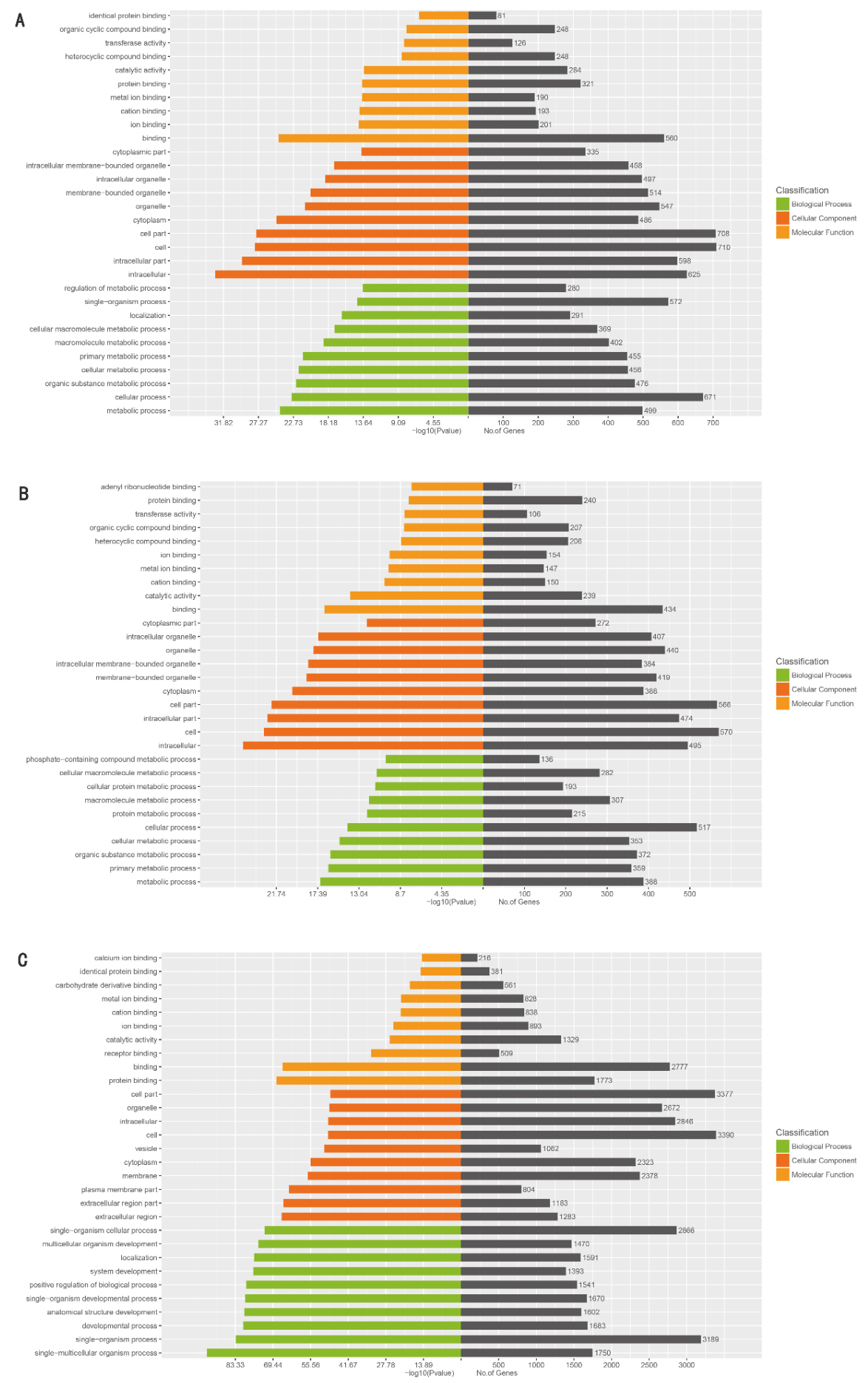


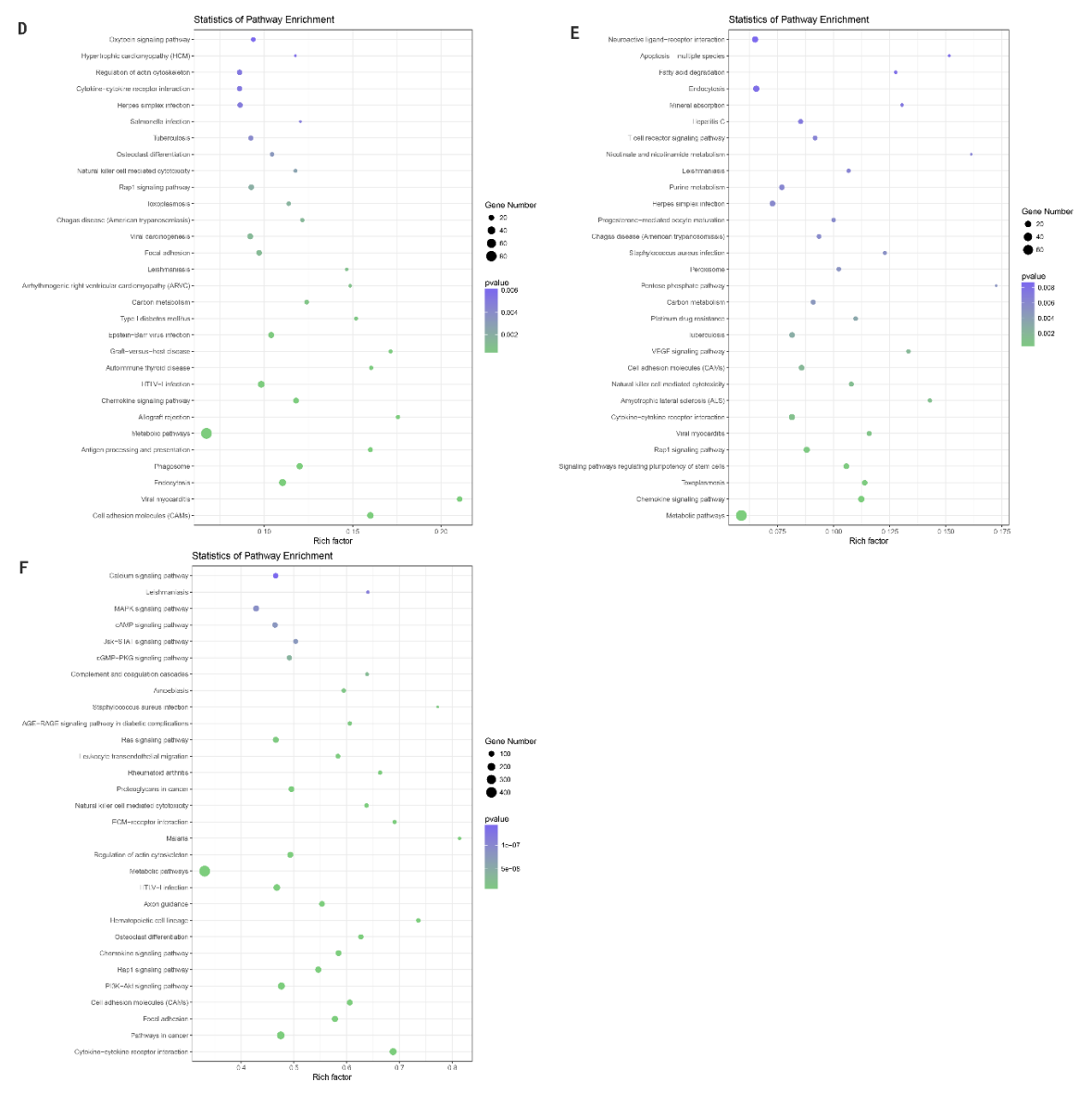



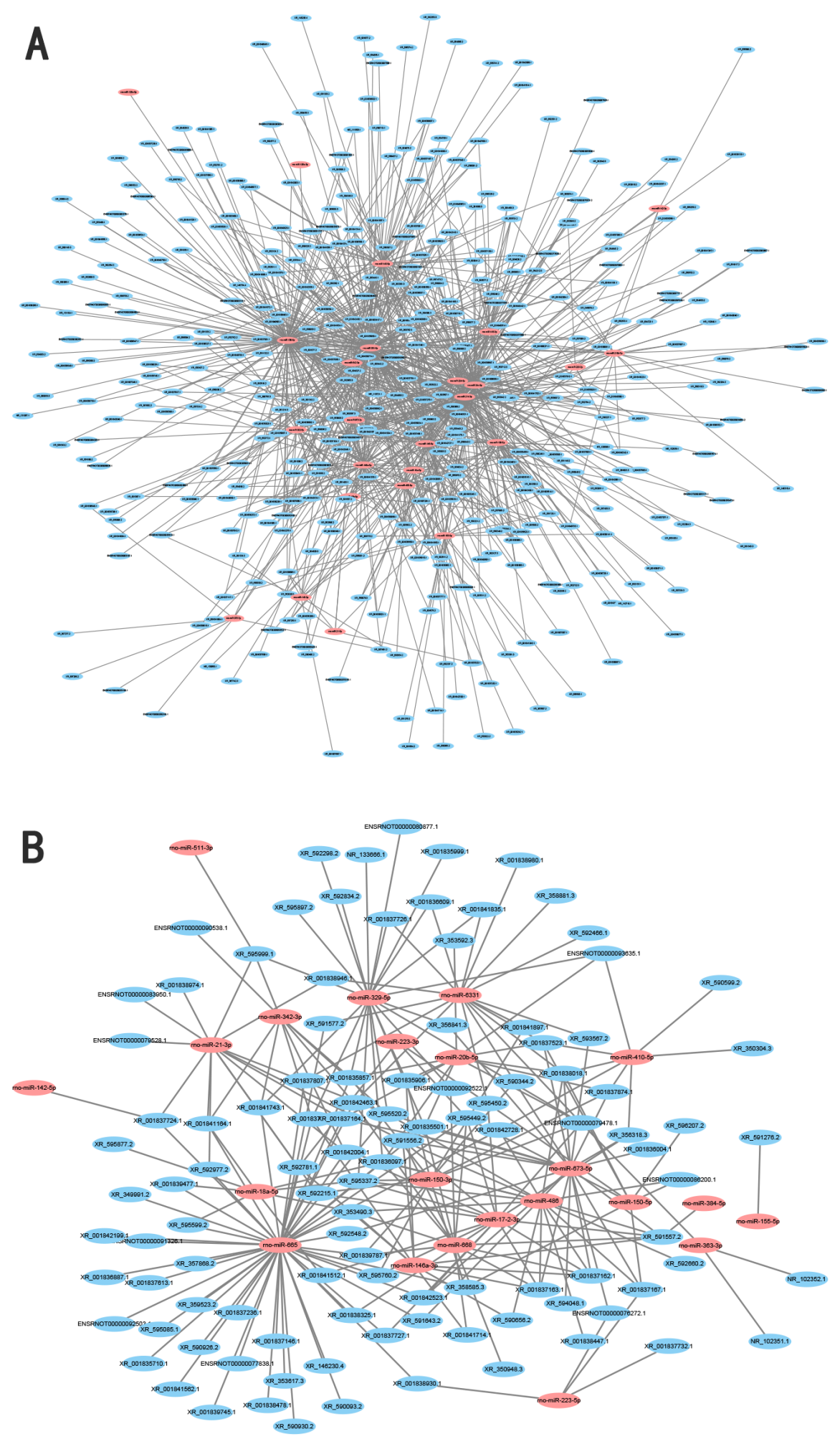

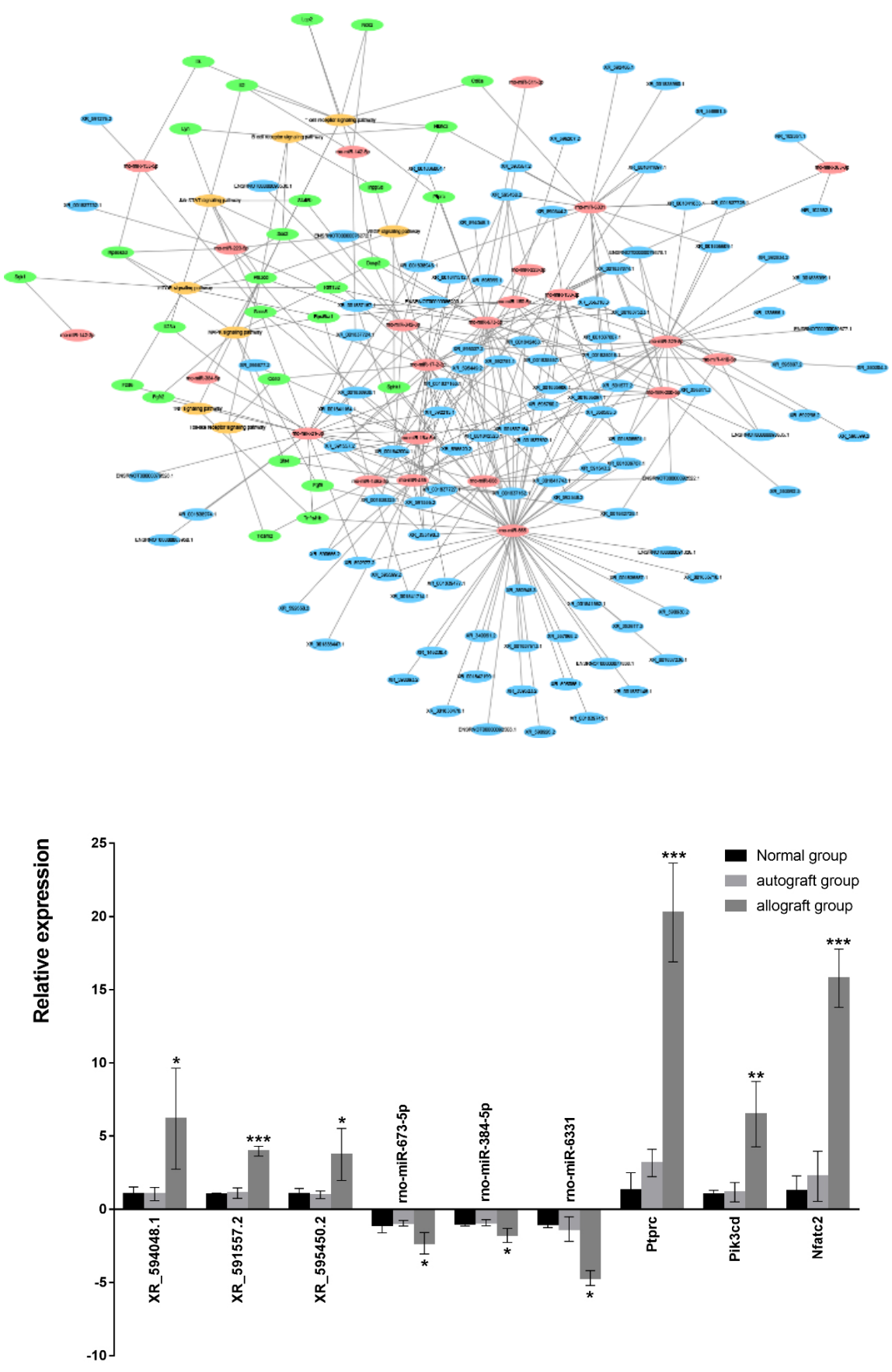